Portland State University

PDXScholar

$5-25-2018$

\title{
Uncovering Newton's Laws within a Game of Tug-of-
} War

Roberto C. Perez-Franco

Portland State University

Follow this and additional works at: https://pdxscholar.library.pdx.edu/honorstheses Let us know how access to this document benefits you.

Recommended Citation

Perez-Franco, Roberto C., "Uncovering Newton's Laws within a Game of Tug-of-War" (2018). University Honors Theses. Paper 588.

https://doi.org/10.15760/honors.597

This Thesis is brought to you for free and open access. It has been accepted for inclusion in University Honors Theses by an authorized administrator of PDXScholar. Please contact us if we can make this document more accessible: pdxscholar@pdx.edu. 


\title{
Uncovering Newton's Laws Within a Game of Tug-of-War
}

\author{
by \\ Roberto C. Pérez-Franco \\ An undergraduate honors thesis submitted in partial fulfillment of the \\ requirements for the degree of \\ Bachelor of Science \\ in \\ University Honors \\ and \\ Physics
}

Thesis Adviser

Ph.D. Ralf Widenhorn 


\section{Table of Contents}

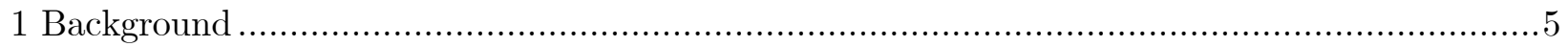

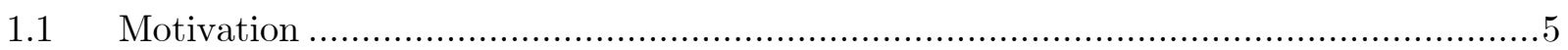

$1.2 \quad$ Newton Laws within Tug-of-War ........................................................................

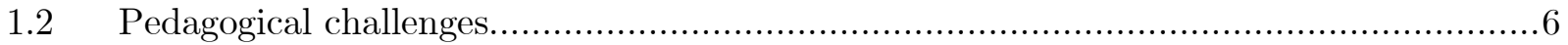

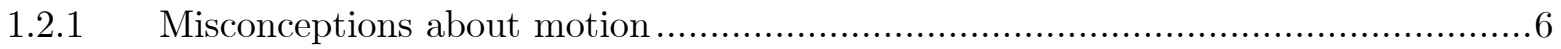

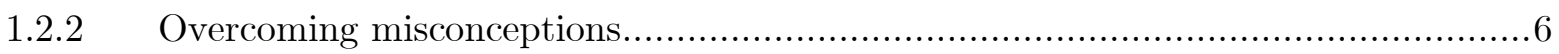

1.2.3 Addressing misconceptions with Tug-of-War......................................................

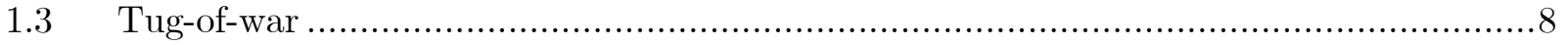

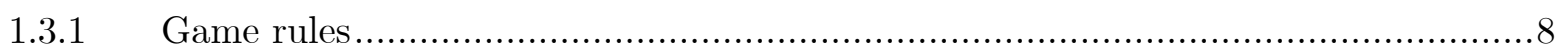

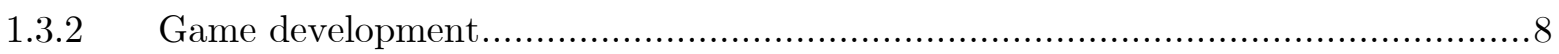

1.3.3 Other factors that influence the game outcome …….........................................

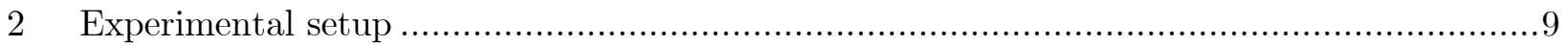

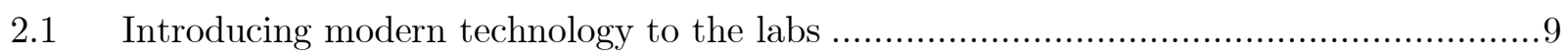

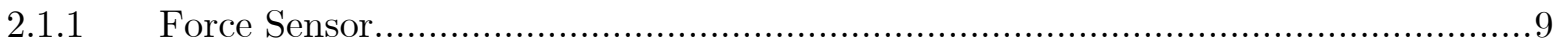

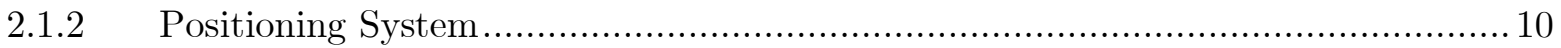

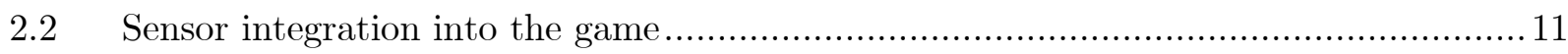

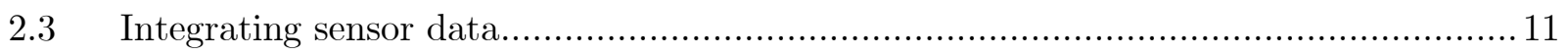

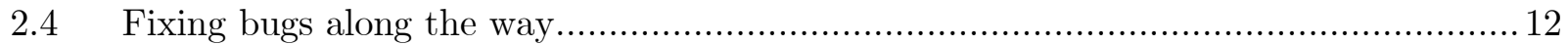

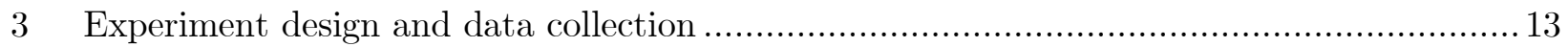

3.1 What do we expect from the matches? ................................................................... 13

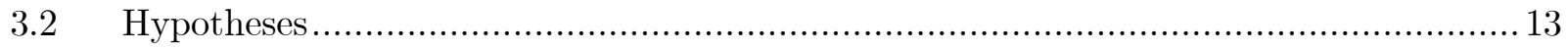

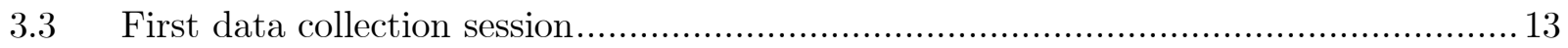

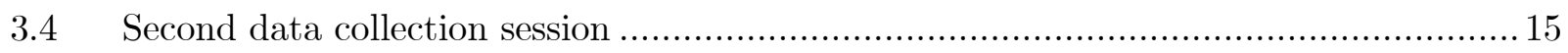

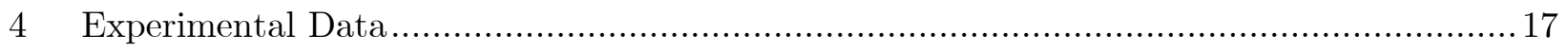

4.1 First data collection session............................................................................. 17

4.1 .13 vs. 2 .

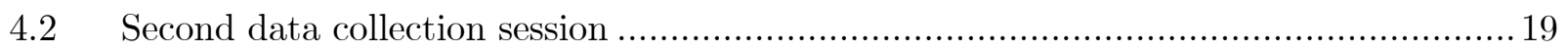

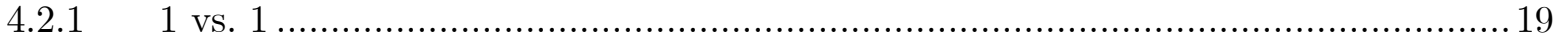

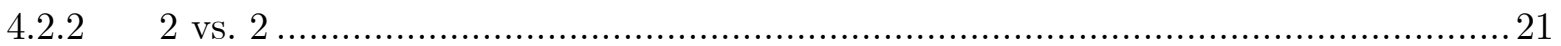

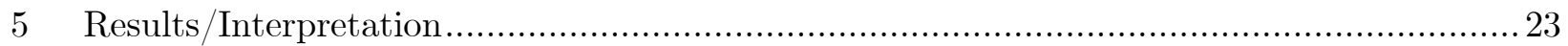




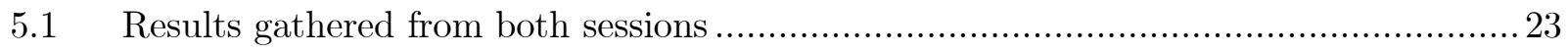

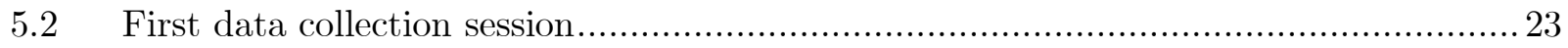

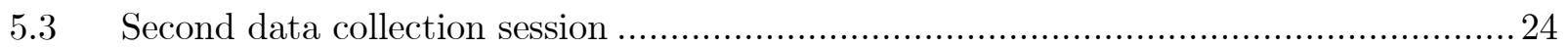

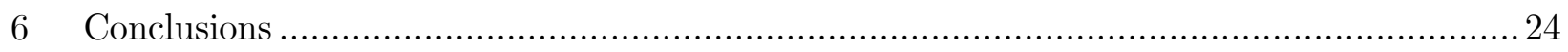

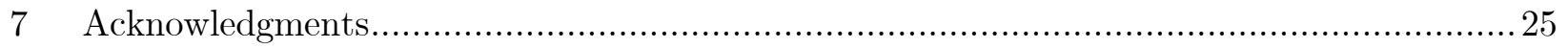

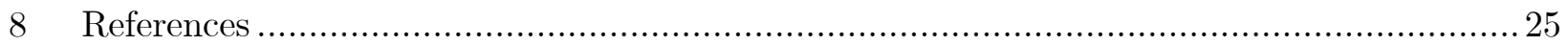




\section{Abstract}

Introductory physics students frequently struggle with the application of Newton's laws of motion. Students frequently misinterpret Newton's $3^{\text {rd }}$ Law, which states that for every action there is an equal and opposite reaction. The confusion arises when allocating the action-reaction force pair to a system; students think that the force pair act on the same object, but the force pair actually act on different objects. One interesting example, where action-reaction force pairs come into play, is the game of Tug-of-War. Therefore, to address some misconceptions about Newton's laws of motion, we implemented an activity where we looked at how the magnitude of the tension force in a game of Tug-ofWar changed over time and what factors lead one team to victory. Using an experimentally modified game setup, we measured the tension on the rope using a load cell and the position of the center of the rope was tracked using an ultra-wideband positioning system. The data collected from these sensors exhibited decreasing trends in the tension force magnitude as each match concluded. The loss of balance of the losing team caused this trend to develop. To further understand the outcome of complex systems like a game of Tug-of-War, new intuition needs to be build. During this engaging activity, students will learn how to correctly apply Newton's laws of motion. Implementing such activity into introductory level physics lab courses, demonstrations, or science outreach activities, will help students describe other complex systems using Newton's laws of motion. 


\section{Background}

\subsection{Motivation}

Students who are introduced to Newton's laws in high school or college often find them to be counterintuitive. Often students form incorrect ideas and mix up concepts by relating them incorrectly with personal experience ${ }^{1}$. Therefore, it is necessary to introduce Newtonian physics in innovative and interactive ways to address these misconceptions. The goal of this project is to develop an activity in which students can participate in Tugof-war matches, and through the implementation of modern technology students will be able to visualize and understand how Newton's laws govern the motion of systems.

\subsection{Newton Laws within Tug-of-War}

In a game of Tug-of-War, students can understand how a match is won by using Newton's first and second law. These laws state that there will be acceleration if and only if there is a net force applied to the system, and that the net force is defined as the sum of all external forces acting on the system. For instance, in the system depicted by the free body diagram in Figure 1, there are two players pulling in opposite directions. In the diagram, the direction of the positive acceleration, a, is to the right. When both players apply equal in magnitude and opposite in direction forces to the ground they will not accelerate because the net force on the system will equal to zero. In other words, $a=0$ when,

$$
\left|\mathrm{F}_{\mathrm{A}}\right|=\left|\mathrm{F}_{\mathrm{TA}}\right|=\left|\mathrm{F}_{\mathrm{TB}}\right|=\left|\mathrm{F}_{\mathrm{B}}\right|
$$

where $\mathrm{F}_{\mathrm{A}}$ is the frictional force on player $\mathrm{A}$ exerted by the ground, $\mathrm{F}_{\mathrm{B}}$ is the frictional force on player $\mathrm{B}$ exerted by the ground; $\mathrm{F}_{\mathrm{TA}}$ and $\mathrm{F}_{\mathrm{TB}}$ are the tension forces applied to the force sensing load cell. However, when the forces are unbalanced, the system accelerates in the direction of the net force. For instance, there will be an acceleration to the right, i.e. a $>0$ when,

$$
\left|\mathrm{F}_{\mathrm{A}}\right|<\left|\mathrm{F}_{\mathrm{TA}}\right|=\left|\mathrm{F}_{\mathrm{TB}}\right|<\left|\mathrm{F}_{\mathrm{B}}\right|
$$

and there will be an acceleration to the left, i.e. $\mathrm{a}<0$ when,

$$
\left|\mathrm{F}_{\mathrm{A}}\right|>\left|\mathrm{F}_{\mathrm{TA}}\right|=\left|\mathrm{F}_{\mathrm{TB}}\right|>\left|\mathrm{F}_{\mathrm{B}}\right|
$$

During the activity, third law force pairs are always equal and opposite to each other, even when players accelerate. The acceleration is caused by the imbalance of frictional forces at different parts of the system. 

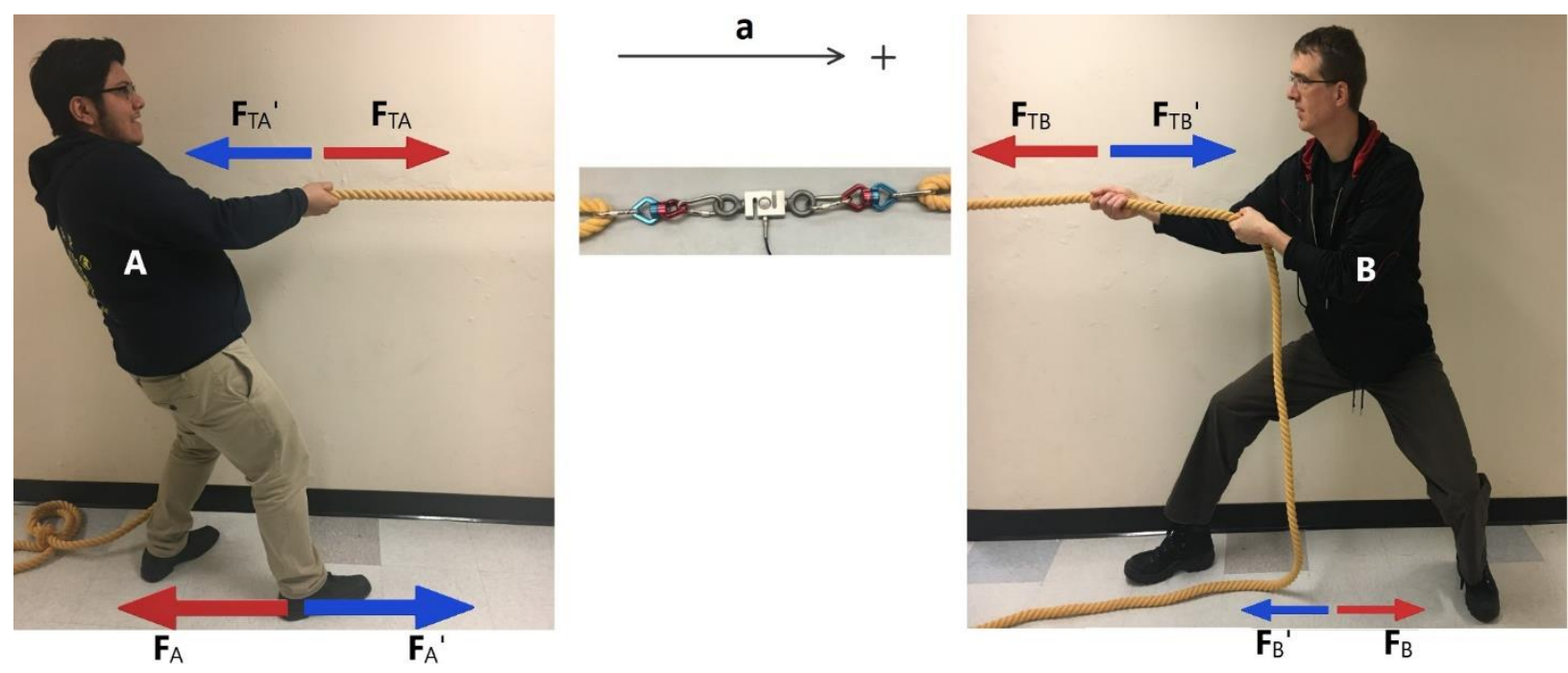

Figure 1. Free body diagram of a game of Tug-of-War between two players. In this match, player A (left) is gaining the upper hand against player B (right) as represented by the size of the force arrow vectors. The positive acceleration, a, is defined to be towards the right. The tension force magnitudes are measured using a load cell placed between the two players. The third law pair forces from left to right are: $\mathrm{F}_{\mathrm{A}}$ and $\mathrm{F}_{\mathrm{A}}{ }^{\prime}$; $\mathrm{F}_{\mathrm{TA}}$ and $\mathrm{F}_{\mathrm{TA}}{ }^{\prime} ; \mathrm{F}_{\mathrm{TB}}$ and $\mathrm{F}_{\mathrm{TB}}{ }^{\prime}$; and, $\mathrm{F}_{\mathrm{B}}$ and $\mathrm{F}_{\mathrm{B}}$ '. Where, $\mathrm{F}_{\mathrm{A}}$ is the force from the ground on player $\mathrm{A}$ and $\mathrm{F}_{\mathrm{A}}$ ' is the force on the ground by player $\mathrm{A}$. Next, $\mathrm{F}_{\mathrm{TA}}$ is the force from the rope on player $\mathrm{A}$ and $\mathrm{F}_{\mathrm{TA}}$ ' is the force on the rope by player A. Similarly, $\mathrm{F}_{\mathrm{TB}}$ is the force from the rope on player $\mathrm{B}$ and $\mathrm{F}_{\mathrm{TB}}$ ' is the force on the ground by player $\mathrm{B}$. Lastly, $\mathrm{F}_{\mathrm{B}}$ is the force from the ground on the player $\mathrm{B}$ and $\mathrm{F}_{\mathrm{B}}$ ' is the force on the ground by player B. The third law pairs are always equal and opposite to each other even when there is movement in the system. Also, note that the tension force vectors relate as follows: $\mathrm{F}_{\mathrm{TA}}=$ $\mathrm{F}_{\mathrm{TB}}{ }^{\prime}=-\mathrm{F}_{\mathrm{TB}}=-\mathrm{F}_{\mathrm{TA}}{ }^{\prime}$.

\subsection{Pedagogical challenges}

\subsubsection{Misconceptions about motion}

There are several misconceptions and erroneous ideas about motion when students first encounter Newton's laws of motion. A questionnaire, called Force Concept Inventory ${ }^{2}$, assessed students on kinematic physics and the results from this test helped the authors develop a list of misconceptions about motion. As stated by the authors of the Force Concept Inventory, addressing each misconception would take a long time. Therefore, it is better to demonstrate to students how Newton's laws describe the motion of objects.

\subsubsection{Overcoming misconceptions}

While memorizing formulas and definitions ${ }^{3}$ can get a student through an exam, an experiment can strengthen one's intuition about natural phenomena. Also, the use of a free body diagram is the principle technique for students to apply Newton's laws of motion because all the forces that act on a system can be labeled. This can help visualize 
the force vectors for the entire system. When students examine only a portion of the system, they can mix concepts from the three laws. For instance, when an object is not accelerating, the second law ${ }^{i}$ and the third lawii can be mixed up. Consequently, a problem where students allocate third law force pair to a single object is prone to emerge. Therefore, students need an activity in which active-learning is applied, i.e. students learn by doing a physical activity or by taking part in an experiment. Allowing students to actively participate in engaging activities like a game of Tug-of-War will help them develop their intuition about motion.

\subsubsection{Addressing misconceptions with Tug-of-War}

The Tug-of-War system is complex due to its several moving parts, but it also shares similarities with other simple phenomena e.g. pulling a box across the floor. Generalizing and thinking that all systems will behave similarly can be detrimental to the understanding of complex systems as misconceptions about the motion of the system will arise. Therefore, in this section, we will focus some of the insightful ideas which will help shed some light on some common misconceptions within the game.

Misconception: The motion of an object is impeded by friction.

While it is true that friction can slow down a moving object, friction ultimately comes down to the interaction between two surfaces. Therefore, it can also be thought of as a force that produces traction. Tug-of-war is a game that uses friction as the driving force.

Misconception: Students often misidentify the action-reaction pair forces described by the third law. They incorrectly identify the action-reaction pair as being the force on a single mass when they are equal and opposite ${ }^{4}$.

Though it is true for equation (1) that the forces are equal in magnitude and opposite in direction, when the players are at equilibrium, the pairing of those forces is incorrect. In other words, pairing the tension force $\left(\mathrm{F}_{\mathrm{TA}}\right)$ with the friction force $\left(\mathrm{F}_{\mathrm{A}}\right)$ on person $\mathrm{A}$ would be an example of an incorrect application of Newton's third law. Being part of Tugof-War games can help develop intuition about Newton's third law. The pair of equal and opposite forces act on different masses e.g. the friction force on person A by the ground $\left(\mathrm{F}_{\mathrm{A}}\right)$ and the friction force on the ground by person $\mathrm{A}\left(\mathrm{F}_{\mathrm{A}}{ }^{\prime}\right)$ would be a third law pair.

\footnotetext{
i Newton's second law: The acceleration of an object is proportional to the net force on an object.

ii Newton's third law: Every action force has an equal and opposite reaction force, where the actionreaction forces act on different objects.
} 
The motion of the system does not affect the third law force pairs; they remain equal and opposite.

Misconception: To move a heavy object, one needs to push harder and harder until the push "overcomes" the resistance and less effort is needed to maintain the motion ${ }^{1}$.

The statement above seems correct at first glance and can be true while pulling a box across the floor. However, generalizing this idea for all systems can lead to labeling systems without thinking through what really is going on, especially in complex systems. For instance, in Tug-of-War, one may think that a team can only win by exerting the greater force throughout the match. Unlike the 'pulling a box' example, the Tug-of-War system has an extra level of complexity, where the opposing team can counter the initial pull by planting their feet on the ground and pulling the other way themselves.

Therefore, to intuitively predict the outcome of a Tug-of-War match it is critical to think through all the variables that make up the system. In later sections the factors that affect the outcome of the game will be presented. In the experiment design section, hypotheses are formed which take these factors into consideration. Then, it will become apparent how a team wins the match. Furthermore, students will be able to predict a match's outcome when given a set of initial conditions about the system. ${ }^{5}$ And vice versa, students will be able to come up with situations or initial conditions in which a team is able to win by maintaining a constant tension force, by causing the other team to exert less force, or by exerting more and more force.

\subsection{Tug-of-war}

\subsubsection{Game rules}

As the goal of the activity is to teach students how to apply Newton's laws into the system, the rules of Tug-of-War are adjusted for a laboratory activity. During the matches players will select even teams at random, so that they are as balanced as possible. Team members can be interchanged if there is a noticeable advantage. The teams will have time to choose a strategy before the match starts. Then, students in the front of each team will have to stand four meters from the center point to start the match. The team that manages to pull the other team over the center will win the match.

\subsubsection{Game development}

The game consists of several stages in which two teams pull on a rope in opposite directions. The first stage consists of developing a posture, either defensive or offensive. In the defensive posture the players do not move their feet; whereas in the offensive 
posture the players start to take steps backwards from the moment they are allowed to pull $^{6}$. The second stage consists of holding a posture as long as possible while transitioning to the next stage. The next stage is called driving; this is when the team begins to move the other team towards their side. The techniques that teams employ are important as these will increase the probability of winning ${ }^{7}$. During the game, players can lean back to lower their center of mass. The amount they go down is related to their position within the team's lineup ${ }^{8}$. A team can also synchronize their pulls ${ }^{9}$ to substantially unbalance the other team.

\subsubsection{How do these techniques influence the force magnitude?}

Maintaining a firm posture in the beginning can influence the tension force magnitude such that it will increase until one team is no longer capable of maintaining their initial frictional grip with the ground. This can be caused by fatigue and/or by the imbalance of players within the team. On the other hand, if the team starts by moving their feet, they will have less friction and a higher chance of losing balance, thus, decreasing the force magnitude applied to the rope. Having a lower center of mass increases stability which, as mentioned before, is related to the greater traction and pulling force. Pulling in unison increases magnitude force such that it reaches its maximum in a match.

\subsubsection{Other factors that influence the game outcome}

Another factor that affects the magnitude of the force is described by the Ringlemann effect ${ }^{10}$. This effect becomes apparent within team activities, as it is the

predisposition of team members to underperform as the number of players involved in the activity increases. In this case, the increase in the number of players creates incoordination within the team which ultimately causes the forces to vanish quicker.

\section{Experimental setup}

\subsection{Introducing modern technology to the labs}

The setup for this activity contains two sensors. One measures force and the other measures position. Both sensors can be set to operate at the same rate, which allows data collection to be synchronized. Therefore, the magnitudes of tension force applied over time and the position of the rope over time can be analyzed together. The details about the force sensor are in section 2.1.1; and the positioning system details are in section 2.1.2.

\subsubsection{Force Sensor}

\subsubsection{Load cell and OpenScale board}

The first component of the force sensor is a load cell which takes mechanical inputs and translates them into proportional electrical signals. The second component of the force 
sensor is an integrated board called OpenScale ${ }^{11}$. This board takes the electrical signals generated in the load cell and processes them to into calibrated force measurements. The load cell output cord is plugged into the OpenScale input (see Figure 2) and the OpenScale output is plugged into a computer that allows users to calibrate the sensor and store pertinent data, more details about the storing process will be explained in section 3 'Experiment design and data collection'.

\subsection{Calibration}

The force sensor needs to be calibrated in order to display accurate force measurements. The calibration process takes place in the Arduino interface (serial monitor); it consists of changing a 'calibration factor' within the OpenScale settings. The calibration can be done in real time because the force readings are displayed continuously on the serial monitor and can be altered until it matches a known force value. In this case, the force sensor was calibrated using water jugs of known mass. The mass of the jugs was measured using a digital scale. Being able to present reliable data is important, therefore the sensor was tested for accuracy before each data collection session.

\subsubsection{Positioning System}

\subsubsection{Pozyx Arduino shields}

The positioning system consists of six Pozyx ${ }^{12}$ devices (see Figure 2). The Pozyx devices are also integrated boards that communicate with other Pozyx devices using ultrawideband signals. Four devices are set as 'anchors'. The anchors are fixed to a position and provide a three-dimensional space in which another Pozyx device, called 'the tag'. These devices can be powered with a rechargeable battery. Such feature allows them to be mounted on any object that needs tracking In this case the tag will be placed in the middle of the rope and its three-dimensional position will be tracked throughout a game.

\subsection{Configuration}

Configurating the positioning system allows for the center of the rope to be accurately located. Therefore, one of the anchors is set to be the origin of the threedimensional space. While the other three anchors can be positioned at known coordinates from that origin. Therefore, the anchor set to be the origin would be at coordinates $(0,0,1000)$, where the $\mathrm{x}$ and $\mathrm{y}$ coordinate are set to be zero and the $\mathrm{z}$ coordinate is measured to be $1000 \mathrm{~mm}$. The $\mathrm{z}$ coordinate is the elevation of the anchor, in this setup the anchor was placed in a tripod one meter above the ground. A three-dimensional 
positioning program $\mathrm{m}^{\mathrm{iii}}$ is used to input the measured coordinates in millimeters $(\mathrm{mm})$. The program is then uploaded to the Arduino board, which has the last Pozyx device plugged into it as a shield.
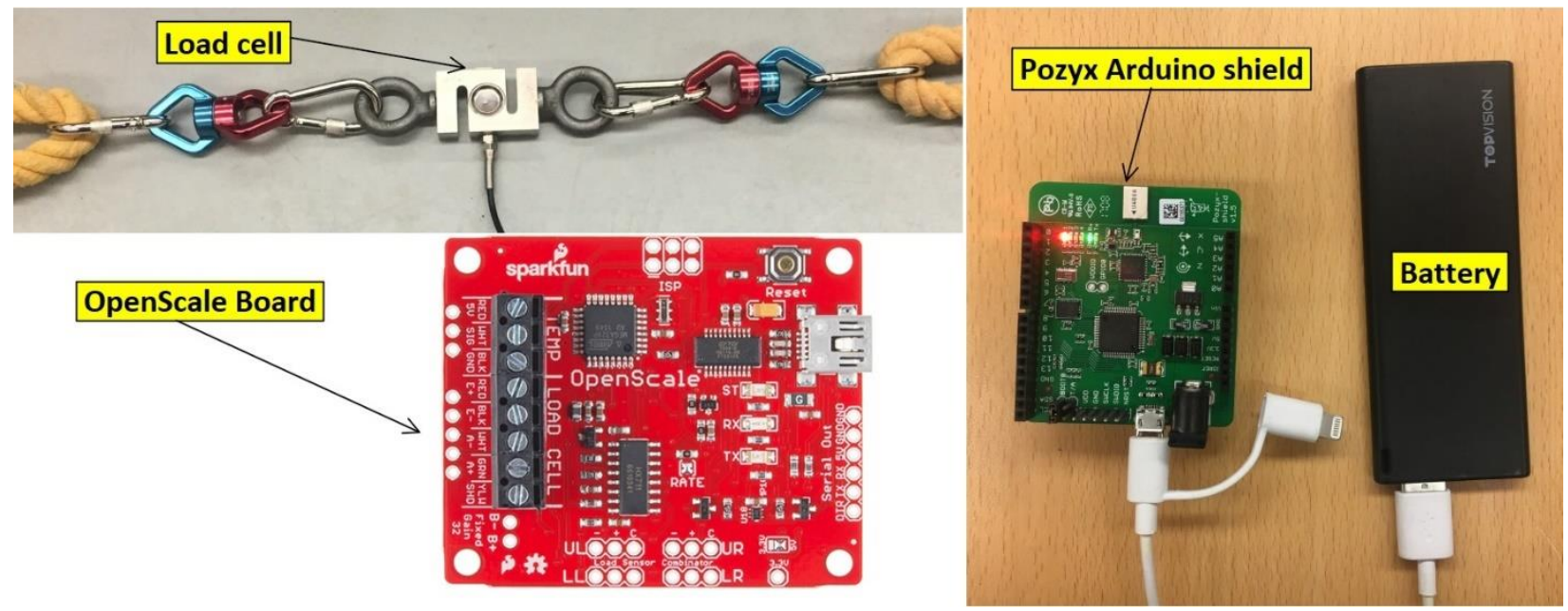

Figure 2. Force sensor and positioning devices. Force sensor: Load cell and OpenScale board. The Pozyx device above is a tag powered by a battery. Five other devices are used to complete the positioning system shown in Figure 3.

\subsection{Sensor integration into the game}

Having set up each of the sensor's software, it is necessary to incorporate each sensor into the game. Placed between the ropes is the calibrated load cell which is plugged into the OpenScale board to collect tension force magnitude data that is stored in a computer. On the other hand, the positioning system works in unison to pinpoint the location of the tag. The tag, powered by a battery, is secured to the rope next to the load cell. The center of the rope position data is then sent to the computer as well. The cartoon version of the setup is shown in Figure 3. The force and position measurements are synchronized timewise to visualize events within the game simultaneously. The next section (2.3) will go into more detail about the program used to synchronize the data.

\subsection{Integrating sensor data}

Since both integrated boards operate in the basis of USB or serial port communication, a program was written to integrate the data incoming from the force sensor and the positioning system. Consequently, to synchronize both sensors, the data

\footnotetext{
iii The Arduino program used to operate the devices can be found in the manufacturer open source repository.
} 
rate from both sensors needed to match. Fortunately, the OpenScale settings contain a data rate calibration option, located in the serial monitor menu, which can be changed accordingly. Once the data rates matched, the force and position measurements were intercalated such that every row in a comma separated value (csv) file represented a moment in time. Therefore, the structure of the program consisted of storing a time stamp with its corresponding force and position data points.

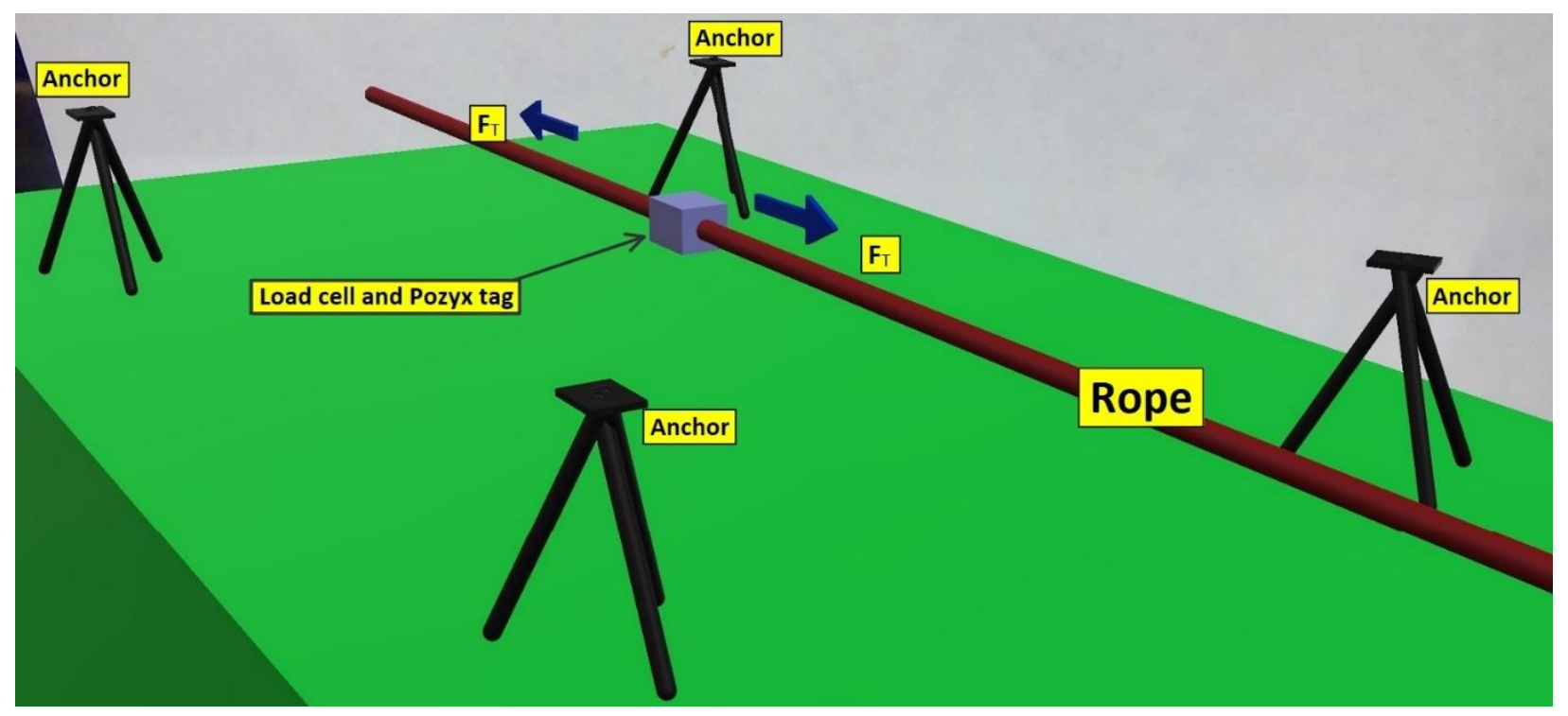

Figure 3. Force sensor and position system setup. The load cell is secured between two ropes while the OpenScale board collects force data. The positioning system consists of six boards: four placed at a fixed distance (anchors) while one (tag) is secured to the center of rope and the last collects position data. The devices collecting data are not in the diagram as they are plugged to a computer that stores both data sets. The force arrow vectors, $\mathrm{F}_{\mathrm{T}}$, represent the tension force applied to the load cell from pulling the rope.

\subsection{Fixing bugs along the way}

As with any experiment development process, there are failed attempts that contribute to further developing the experimental setup. In this case it only took one bad data collection session to address the problems within the setup. Since the load cell has screw eyes to attach the rope to either side, these screws would become loose because of the rotational motion generated as teams pulled. Shown in Figure 4 is one of the swivels that solved this problem. 


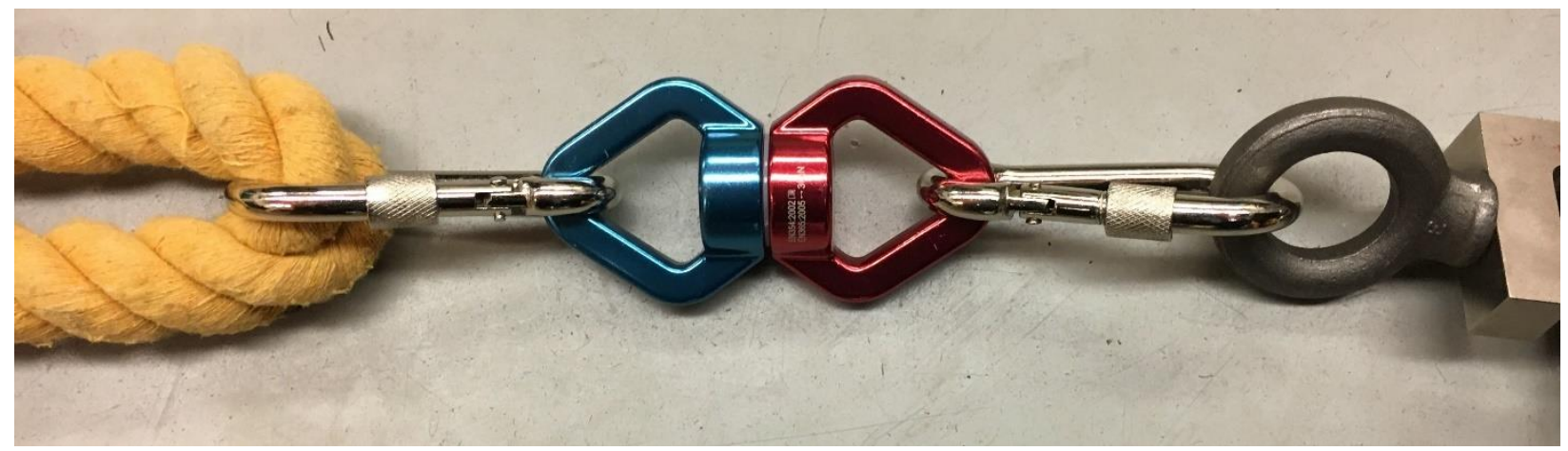

Figure 4. Swivel used to prevent detaching of rope from load cell.

\section{Experiment design and data collection}

\subsection{What do we expect from the matches?}

There are several reasons/motivations for collecting data and presenting data, rather than just presenting the setup of the activity. For one, the current literature about the game discusses techniques and strategies that help teams win, however, there is no data that shows a complete force over time development. On the other hand, it is necessary to test the equipment to see how it would work for students if this activity were to be implemented in a didactic environment like a general physics laboratory. In general, testing the setup provides insight into what students can take away from this activity, be it concept clarification or intuition building.

\subsection{Hypotheses}

First, we hypothesized that the tension force magnitude will decrease over time within a match of Tug-of-war, therefore we did two data collection sessions that were different from each other to see if the hypothesis would hold, regardless of the circumstances. During each session, we also tested for other hypotheses. In one session, we tested for our second hypothesis: the proportionality between frictional force and tension force within the system. While in the other session, we tested for our third hypothesis: the relation between the in maximum tension force exerted by individual players and the maximum tension force of a team composed of these individual players.

\subsection{First data collection session}

As mentioned in the second hypothesis, we wanted to see if the coefficient of friction was reflected on the tension force magnitude. Since the friction depends on the interaction between two surfaces, the matches for this session consisted on varying the interacting surfaces. There were two factors tested. First, the material of the surface was changed. In 
one match a team used shoes and in the other the same team used socks. Second, having different points of contact between the surfaces can also disturb the interaction between them, therefore, both teams were asked to switch to their less dominant position. ${ }^{\text {iv }}$ To test this claim, 3 vs. 2 matches were set up.

In this case the anchors needed to cover an area of eight meters by four meters, satisfying the Tug-of-War rules which indicate that there needs to be eight meters between the first player of each team, and providing enough space to avoid hitting the anchors while moving backwards. Since we are measuring the relevance of the coefficient of friction, it is important to note that the floor where these matches took place was polished wood.

Four matches were conducted between a team of three players and a team of two players. The team of three competed in bare feet during the four matches. The team of two alternated between wearing shoes and socks. In the other two matches both teams switched to their less dominant position. Since the team of three did not change their coefficient of friction, the matches will be described by the team of two for the following text and figures. For example, when the team of two wore shoes the corresponding label would be '3v2 shoes'.

The first match was '3v2 shoes'. Unfortunately, there was a discrepancy as to which team won the match, as the middle of the rope ended near the starting point by the end of the match and both teams gave up on pulling. Therefore, this match's data will not be included as part of the analysis as it did not develop a winner. Nevertheless, including this as part of the activity will be important because students can learn from comparing matches with different coefficients of friction. The second match was '3v2 socks'. The team of three won this match. During this match the team of three was able to pull the team of two with ease as the team of two slid across the floor at a steady pace. The team of two was not able to make any sort of recovery during the sliding process.

The third match was '3v2 shoes' and both teams were pulling from their less dominant side. The team of two won this match. There was no sliding at all from the team of three. The displacement happened when the upper body of the team of three members would move forward and their feet would move quickly to try to regain balance. The fourth match was ' $3 \mathrm{v} 2$ socks' and both teams were pulling from their less dominant side. The team of three won this match as well. The match started with the team of two winning, this was mainly because one member of the team of three lost their balance. However, after they regained balance, the team of three was able to pull the team of two

\footnotetext{
iv In hindsight, it would have been better to keep the team that did not change their interacting surface in their dominant side, as this would have made the tests more controlled.
} 
at a steady pace. There was some sliding during this match, but not as much as the other match that involved socks. The displacement happened when the team of two lifted their feet after a brief period of sliding. Doing this gave them the chance to regain stability and pull towards their side. This was not enough because when the team of three pulled again the sliding would resume.

While the data for the first match was inconclusive, there is still a teachable lesson about friction within these matches. The team of three won both matches when the other team were wearing shoes. Figures 5, 6 and 7 display how the matches for this data session developed.

\subsection{Second data collection session}

To test the third hypothesis, we used the maximum tension force measured during a match as the main parameter. Part of the hypothesis was also prompted to confirm the claim that the maximum tension force was determined by how much the weaker player/team could pull. The reasoning behind this emerged from the idea that the winning team is analogous to a wall and the losing team is trying to bring the wall down. Therefore, we can say that this wall can resist just enough force to keep itself standing and that the weaker team must had exerted the maximum tension force possible at some point. While a linear relationship would be the most reasonable first approximation, the fatigue factor also determines how much and how long a team can pull, which will undoubtedly influence the maximum tension force. It is also important to note that the floor where these matches took place was linoleum.

The experiment designed to tests test this hypothesis consisted of two kinds of matches. There were individual matches $(1 \mathrm{v} 1)$ and there were team matches $(2 \mathrm{v} 2)$. The 1v1 matches were set up to determine the strongest player among four players. These matches also serve to determine the maximum tension force of the weakest players. After that, the strongest player would pair up with one of the weaker players systematically such that all weak players would pair up with the strongest player to match against the remaining two players in 2v2 matches.

In this case, a distinct configuration of the positioning system was used. The new configuration only consisted of two Pozyx devices instead of six. The only difference was that there were no anchors, so the tag reported its displacement from the Arduino shield, which was plugged to a computer for data collection. Having only two devices restricted 
data collection to one-dimensionv. The Arduino program ${ }^{\mathrm{vi}}$ was different, as it only tracked the distance between the tag and the shield, however, there was no coordinates configuration necessary. The distance measured by the sensor and displayed in the graphs of this data collection session is no the actual displacement but the geometric sum ${ }^{\text {vii }}$ of the height of the rope and the actual distance.

This data set consists of the six matches. There were three $1 \mathrm{v} 1$ and another three $2 \mathrm{v} 2$ matches. As mentioned before, the $1 \mathrm{v} 1$ matches served two purposes, determining the maximum tension force of the weaker players and determining which player was the strongest. On the other hand, the $2 \mathrm{v} 2$ matches serve to compare the sum of individual maximum tension forces against the combined maximum tension force exerted by the two players when pulling as a team. The four players were labeled ' $\mathrm{A}$ ' through ' $\mathrm{D}$ ', player A being the weakest and player $\mathrm{D}$ the strongest. The first 1v1 match was between player $\mathrm{A}$ and player B, player B won. The second match was between player B and player D, player $\mathrm{D}$ won. The last match was between player $\mathrm{C}$ and player $\mathrm{D}$, player $\mathrm{D}$ won. Figures 8,9 and 10 display how these matches developed. After the 1v1 matches were done players were paired for the 2v2 matches. The first match was between team $\mathrm{AD}$ and team $\mathrm{BC}$; team $\mathrm{AD}$ won. The second match was between team $\mathrm{BD}$ and team $\mathrm{AC}$; team $\mathrm{BD}$ won. The third match was between team CD and team AB; team CD won. Figures 11, 12 and 13 display how these matches developed.

\footnotetext{
v Since the motion of the rope is primarily along one axis, the change in position within the other axes can be neglected as they remain fairly constant during this experiment.

vi Also provided in the open source repository.

vii The geometric sum being $a=\sqrt{b^{2}+c^{2}}$.
} 


\section{Experimental Data}

\subsection{First data collection session}

\subsubsection{3 vs. 2}

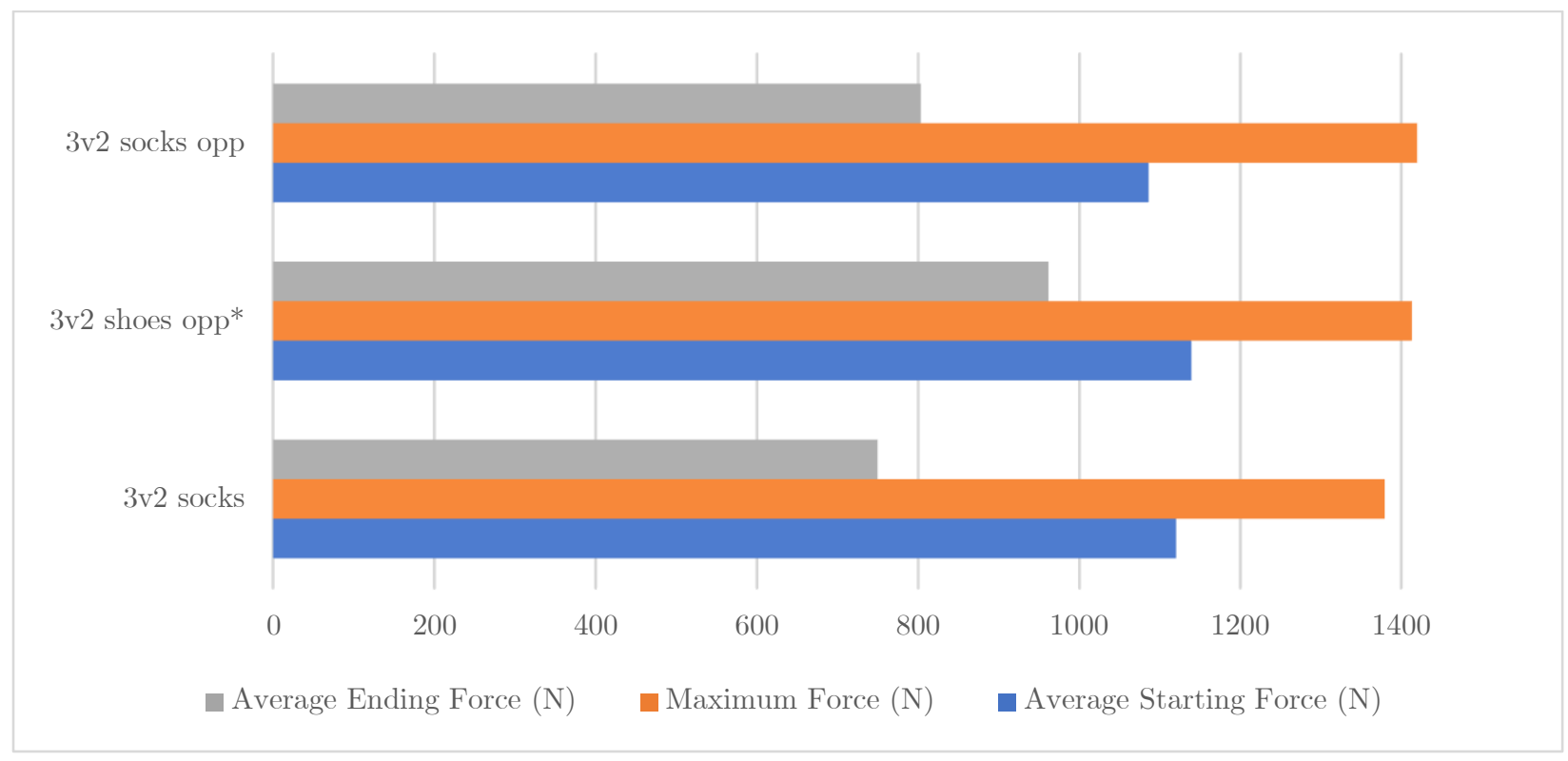

Figure 5. The vertical axis shows data for each of the three matches played. The bars represent forces (in Newtons) at different stages in the match: the blue corresponds to the average starting force, the orange to the maximum tension force achieved, and the gray to the average ending force. There were two teams: one with three members in bare feet, and the other with two members changing between having shoes (not in the graph due to inconclusive winner) or socks on. After these two matches, all team members switched to their less dominant side for another two matches, labeled as 'opp' in the graph. * The only occasion where the team of two won the match. Notice how the starting force is greater than the ending force.

Table 1. Numerical force values from each of the matches in Figure 5. Marked with an $*$ is the match where the team of two won.

\begin{tabular}{|c|c|c|c|}
\hline Matches & Average Starting Force $(\mathrm{N})$ & Maximum Tension Force (N) & Average Ending Force $(\mathrm{N})$ \\
\hline $3 \mathrm{v} 2$ socks & 1120 & 1380 & 750 \\
\hline 3v2 shoes opp* & 1139 & 1413 & 962 \\
\hline 3v2 socks opp & 1086 & 1420 & 803 \\
\hline
\end{tabular}




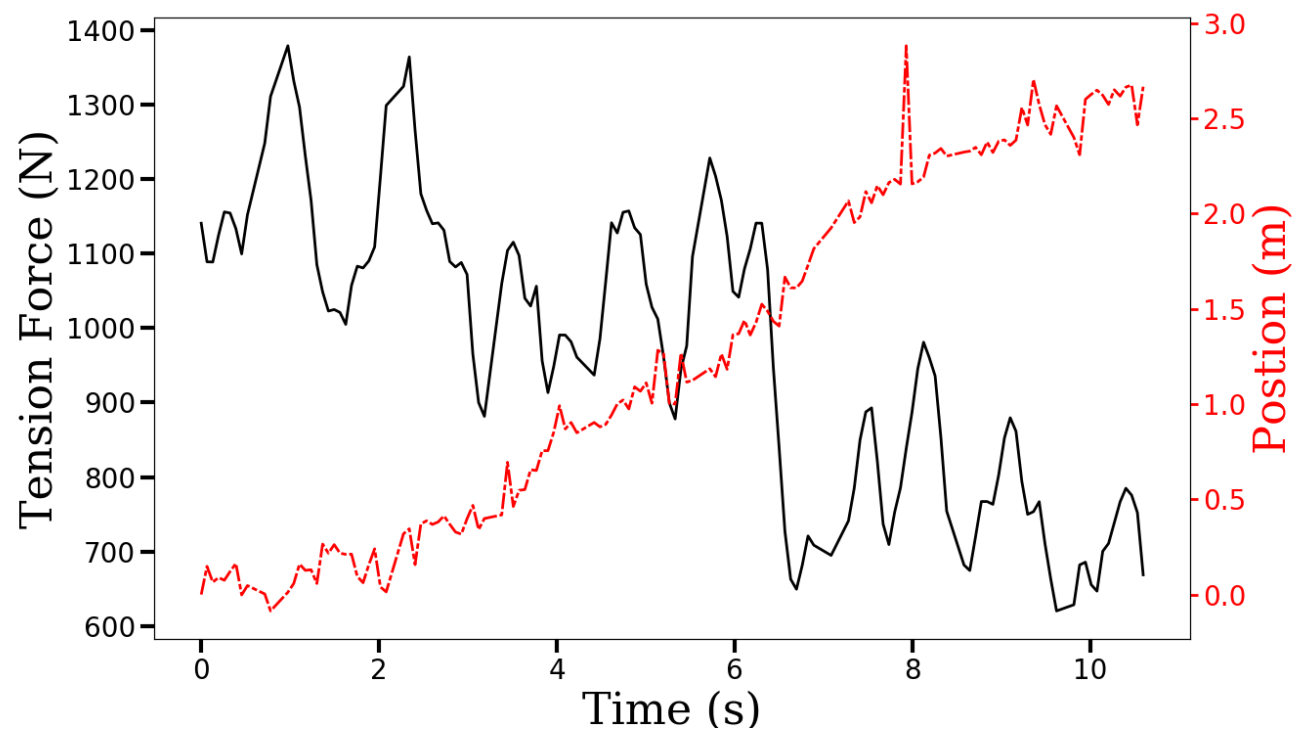

Figure 6. The tension force magnitudes (in Newtons) are on the left vertical axis (solid black) and the position magnitudes (in meters) are on the right vertical axis (dashed red). The time (in seconds) is on the horizontal axis. The data comprises the events of a match in where a team of three people in bare feet wins against a team of two people wearing socks in about 11 seconds. In some instances, the position data was modified so that the last point ends on the upper right corner of the graph, this is the case for all other force and position graphs.
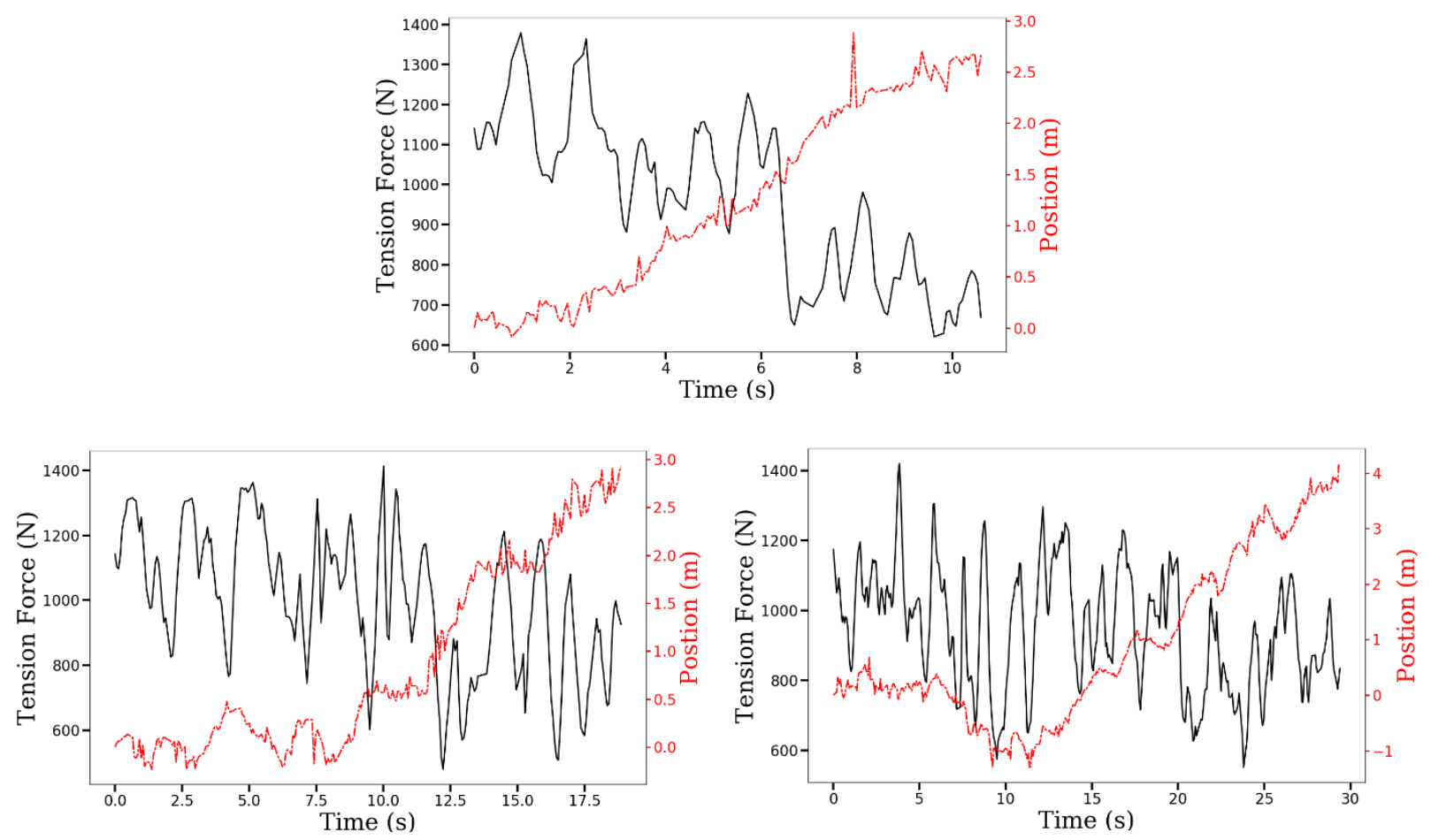

Figure 7 . This set of graphs includes the three $3 \mathrm{v} 2$ matches, which show the general trend of decreasing tension force over time (solid black). On the upper center is the ' $3 \mathrm{v} 2$ socks match. On the lower left is the '3v2 shoes opp' match and on the lower right is the '3v2 socks opp' match. 


\subsection{Second data collection session}

\subsubsection{1 vs. 1}

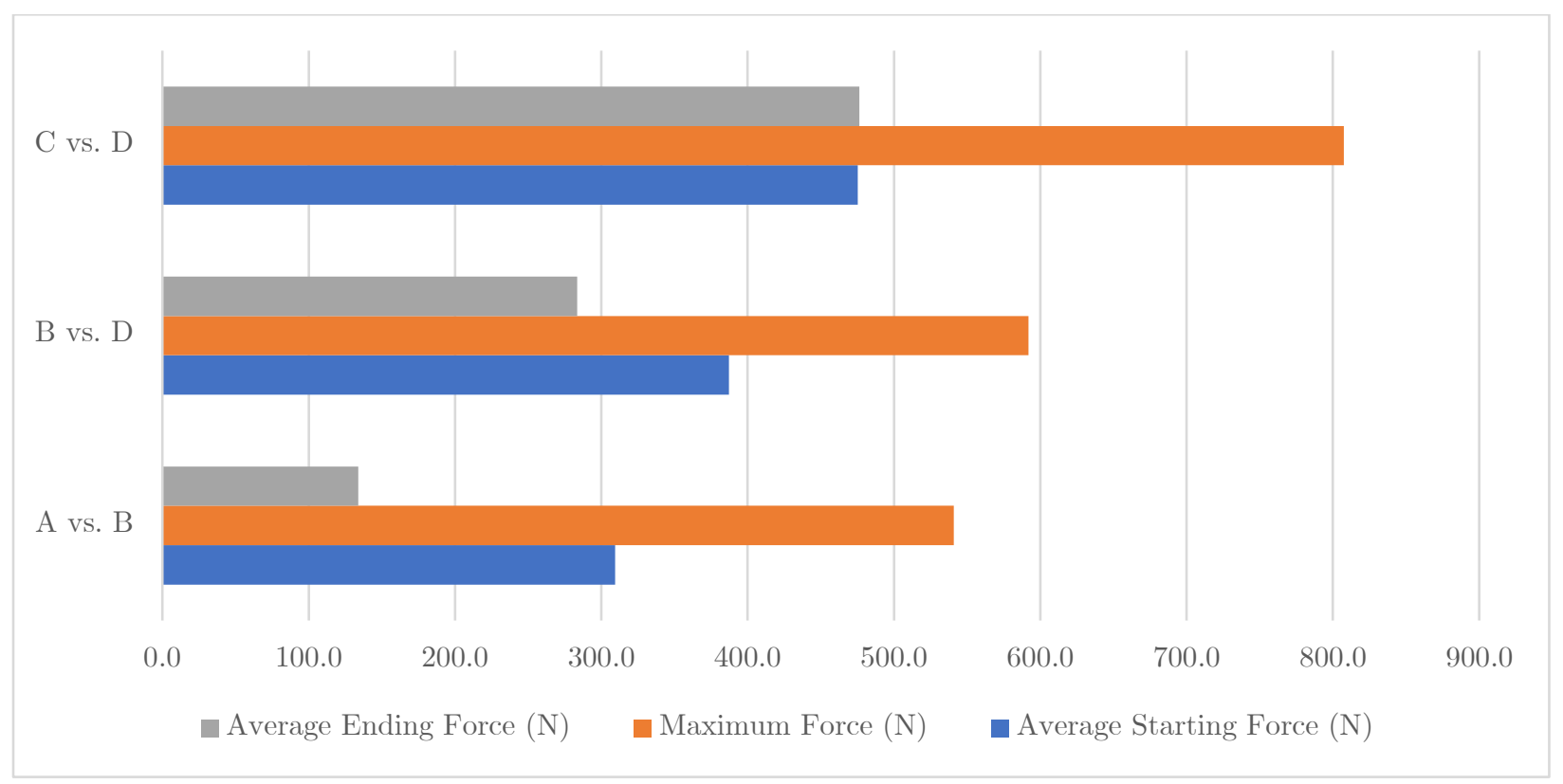

Figure 8. The bars represent forces (in Newtons) at different stages in the match: the blue corresponds to the average starting force, the orange to the maximum tension force achieved, and the gray to the average ending force. Each block of bars represents a 1v1 match. The players were labeled A through D to rank them from weakest to strongest. Therefore, the player on the right was the winner of the match and the player on the left determined the maximum tension force of the math. Notice how the starting force is greater than the ending force.

Table 2. Numerical tension force values from each of the matches in Figure 8. In the $\mathrm{C}$ vs. D match, the average starting force is less than the average ending force

\begin{tabular}{|c|c|c|c|}
\hline Matches & Average Starting Force (N) & Maximum Tension Force (N) & Average Ending Force $(\mathrm{N})$ \\
\hline A vs. B & 309.4 & 540.9 & 133.9 \\
\hline B vs. D & 387.1 & 591.9 & 283.6 \\
\hline C vs. D & 475.2 & 807.5 & 476.3 \\
\hline
\end{tabular}



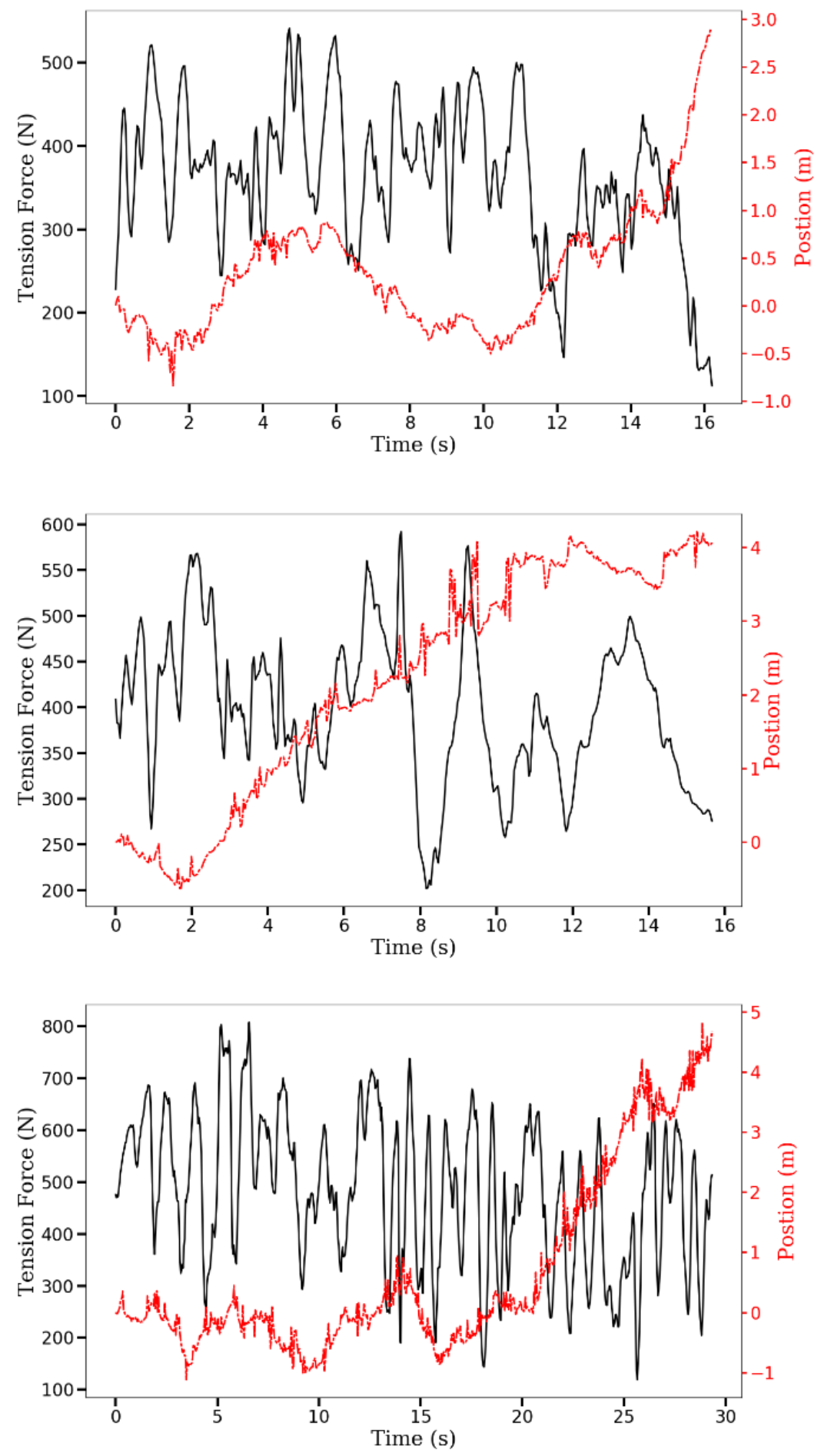

Figure 10. These set of graphs show the tension force in newtons (solid black) and the position in meters (dashed red) for the 1v1 matches. On the top is the A vs. B match. In the middle is the B vs. D match. On the bottom is the $\mathrm{C}$ vs. $\mathrm{D}$ match. The downward trend for the tension force magnitude was not apparent for these matches, especially on the last match that is because the starting force was greater than the ending force, see Table 2 for exact values. Nevertheless, by looking at the peaks of the force the downward trend is visible. 


\subsubsection{2 vs. 2}

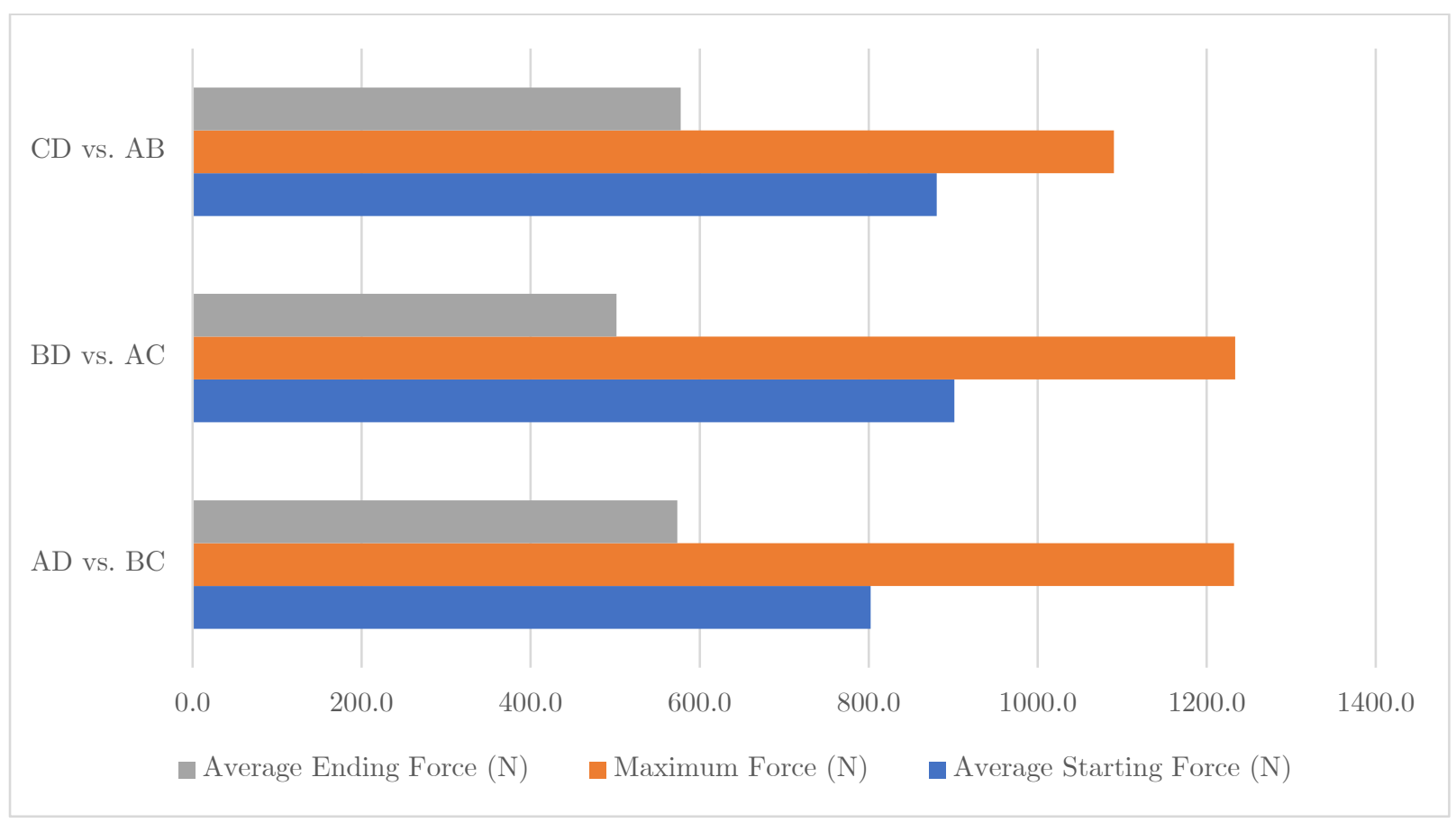

Figure 11. The bars represent forces (in Newtons) at different stages in the match: the blue corresponds to the average starting force, the orange to the maximum tension force achieved, and the gray to the average ending force. Each block of bars represents a $2 \mathrm{v} 2$ match. The players were labeled A through D to rank them from weakest to strongest. Then, the strongest player was systematically paired with the other players. All the teams on the left won the matches which means that the teams on the right determined the maximum tension force for each of the matches. Notice how the starting force is greater than the ending force.

Table 3. Numerical tension force values from each of the matches in Figure 11.

\begin{tabular}{|c|c|c|c|}
\hline Matches & Average Starting Force $(\mathrm{N})$ & Maximum Tension Force (N) & Average Ending Force $(\mathrm{N})$ \\
\hline $\mathrm{AD}$ vs. $\mathrm{BC}$ & 802.1 & 1232.5 & 573.4 \\
\hline $\mathrm{BD}$ vs. $\mathrm{AC}$ & 901.2 & 1233.6 & 501.5 \\
\hline $\mathrm{CD}$ vs. $\mathrm{AB}$ & 880.4 & 1090.1 & 577.6 \\
\hline
\end{tabular}



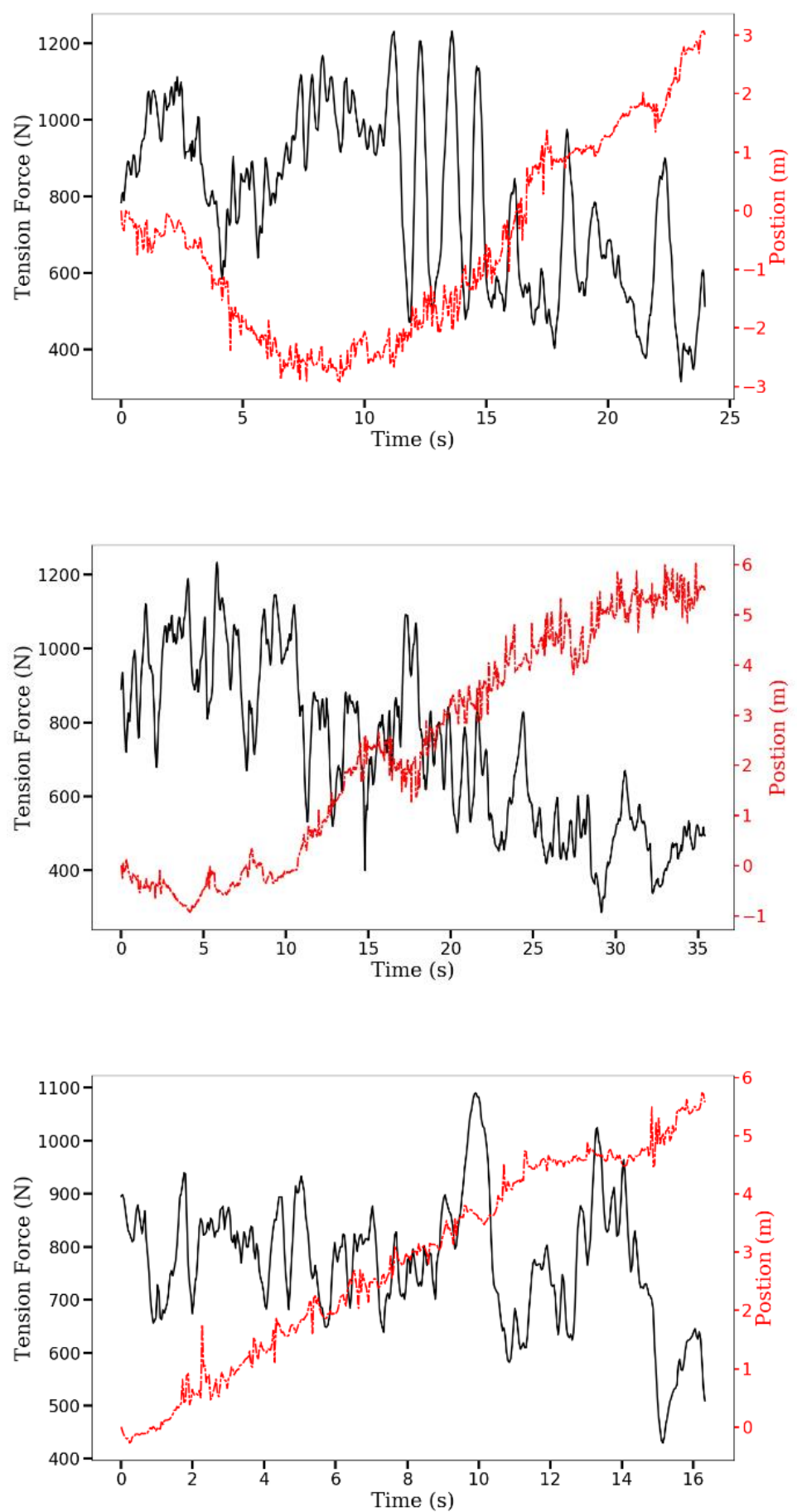

Figure 12. These set of graphs show the tension force in newtons (solid black) and the position in meters (dashed red) for the $2 \mathrm{v} 2$ matches. The players were labeled $\mathrm{A}$ through $\mathrm{D}$ to rank them from weakest to strongest. On the top is the AD vs. BC match. In the middle is the BD vs. AC match. On the bottom is the $\mathrm{CD}$ vs. $\mathrm{AB}$ match. Once again, the downward trend developed in the tension force magnitude. 
Table 4. The individual maximum tension forces of two players were summed and then compared to the maximum tension force of the team composed of these two players. One of the comparisons is the difference between the resulting sum and the team maximum tension force. While the other comparison was a percent difference between those values. These matches only include data of the weaker players as they are the ones determining the maximum tension force in a match.

\begin{tabular}{ccccc}
\multicolumn{2}{c}{ Match } & Maximum Tension Force $(\mathrm{N})$ & Difference & Percent Difference \\
\hline $1 \mathrm{v} 1$ & $(\mathrm{~A}+\mathrm{B})$ & 1132.8 & 42.7 & $4 \%$ \\
$2 \mathrm{v} 2$ & $(\mathrm{AB})$ & 1090.1 & & $9 \%$ \\
\hline $1 \mathrm{v} 1$ & $(\mathrm{~A}+\mathrm{C})$ & 1348.4 & 114.8 & \multirow{2}{*}{$12 \%$} \\
$2 \mathrm{v} 2$ & $(\mathrm{AC})$ & 1233.6 & 167.0 & \\
\hline $1 \mathrm{v} 1$ & $(\mathrm{~B}+\mathrm{C})$ & 1399.5 & & \\
$2 \mathrm{v} 2$ & $(\mathrm{BC})$ & 1232.5 & \\
\hline \hline
\end{tabular}

\section{Results/Interpretation}

\subsection{Results gathered from both sessions}

While analyzing the data, we looked for a trend in the tension force magnitude over time. In both data collection sessions, we found that there was indeed a downward trend in the force magnitude which can be seen in the sets of graphs encapsulated in Figures 7, 10 and 12. The factors that play a role in determining this magnitude decrease trend are many, but among the ones with most apparent relevance are fatigue, friction and balance.

\subsection{First data collection session}

The coefficient of friction was the main parameter tested in this data collection session. While the experiment only accounts for the tension force, being able to see the effect of a lower coefficient of friction in the tension force is a teachable lesson for students. Comparing the time intervals that it took the teams to achieve victory provided further insight into how friction impacts the games development and lets us establish a relation between the tension force and the friction force. For instance, during the match where teams were pulling from their dominant side and one team was wearing socks, it only took 11 seconds to defeat the team in socks. On the other hand, when teams were pulling from their less dominant side, the time intervals varied. When the team of two won with shoes on, it took them 18 seconds. But when they lost while wearing socks, the match lasted for over 30 seconds. These results give reason to believe that friction was influenced by both the material of the surfaces and the way the surfaces interacted. 


\subsection{Second data collection session}

The maximum tension force was the main parameter analyzed for these sets of matches. After comparing the maximum tension force values in Tables 2 and 3; i.e. the maximum tension force displayed by each player in the $1 \mathrm{v} 1$ matches and the maximum tension force displayed in the $2 \mathrm{v} 2$ matches, it was possible to see that the maximum tension force from the individual player matches added up to the maximum tension force in team matches. These results are compared further in Table 4. One of the comparisons done within the table was a subtraction between the sum of two players' maximum tension forces obtained from the 1v1 matches and the maximum tension force displayed by the team composed of these two players from the $2 \mathrm{v} 2$ matches. These results are qualitatively consistent with the Ringlemann effect, as we see a decrease in the maximum tension force in all three comparisons when the individuals join to form a team. On the other hand, the quantitative relevance of the values can be attributed to several aspects with in the game, such as fatigue.

\section{Conclusions}

The research and development stages of this project lead to the insightful results about the outcome of a game of Tug-of-War and to the making of a new and innovative experiment design. Furthermore, it also sprouted the idea of taking the experimental setup developed in a physics classroom. This idea further developed when we looked into the field of physics pedagogy, whereby touching the surface of results and compiled tests done in introductory physics courses, we were able to narrow down misconceptions about newtons laws of motion that applied to the ideas concepts we were trying to understand about the tug-of-was system.

There were some important takeaways as far as winning strategies. For instance, the general technique to win a match is to maintain balance at all costs. The complexity of this system is greater than that of pulling a box across the floor simply because we are dealing with non-rigid bodies connected through a tensed rope. Keeping the upper body close to the ground is a good technique to maintain good stability, as our center of mass changes by altering the shape of our body. Having such stability can influence the footing/friction and lead to victory.

Last but not least, the experimental setup developed for Tug-of-War games during this research, integrates modern technology which offers innovative ways of teaching Newton's laws. Therefore, including this activity in college or high school level physics courses allows students to either confirm or correct their intuition about the motion of complex systems in an engaging manner. 
The appreciation of the author goes out to all the Widenhorn lab members for helping and guiding this project toward its final form. Specifically, Ph.D. Ralf Widenhorn, academic adviser, who proposed the idea of the project and provided both his physics and pedagogy expertise, and Cora Siebert, our lab manager, who organized the data collection sessions and provided her expertise about the positioning system used in the experiment.

\section{References}

${ }^{1}$ I.A. Halloun and D. Hestenes, Am. J. Phys. 53, 1056 (1985).

${ }^{2}$ D. Hestenes, M. Wells, and G. Swackmamer, Phys. Teach. 30, 141 (1992).

3 J. Bruun and F.V. Christiansen, (2014).

${ }^{4}$ D. Low and K. Wilson, Teach. Sci. J. Aust. Sci. Teach. Assoc. 63, 17 (2017).

${ }^{5}$ K. Zetie, 23, 8 (2013).

6 J.-H. Tu, C.-H. Lee, and Y.-H. Chiu, in ISBS-Conf. Proc. Arch. (2008).

7 M. Godfrey, M. Nakagawa, and H. Yamamoto, in ISBS-Conf. Proc. Arch. (2007).

8 N. Ryuji, M. Nakagawa, and H. Yamamoto, in ISBS-Conf. Proc. Arch. (2007).

${ }^{9}$ K. Tanaka, S. Kawahara, N. Minamitani, M. Fukushima, C. Yulin, and H. Yamamoto, in ISBS-Conf. Proc. Arch. (2007).

10 C. Liou, T.-L. Wong, J.-C. Wang, and J.-C. Shin, in ISBS-Conf. Proc. Arch. (2008).

11 OpenScale Website, (https://learn.sparkfun.com/tutorials/openscale-applications-andhookup-guide).

12 Pozyx Website, (www.pozyx.io). 